\title{
Synthesis of Controllable Carboxylated Polystyrene Microspheres by Two-Step Dispersion Polymerization with Hydrocarbon Alcohols
}

\author{
Bin Zhang, ${ }^{1,2}$ Jiazhen Lu, ${ }^{1}$ Xin Liu, ${ }^{1}$ Haibo Jin $\mathbb{D}^{1,2}$ Guangxiang He $\mathbb{D}^{1,2}$ \\ and Xiaoyan Guo $\mathbb{1}^{1,2}$ \\ ${ }^{1}$ College of Chemical Engineering, Beijing Institute of Petrochemical Technology, Beijing 102617, China \\ ${ }^{2}$ Beijing Key Laboratory of Fuels Cleaning and Advanced Catalytic Emission Reduction Technology, Beijing 102617, China \\ Correspondence should be addressed to Guangxiang He; hgx@bipt.edu.cn and Xiaoyan Guo; guoxiaoyan@bipt.edu.cn
}

Received 15 June 2018; Revised 17 August 2018; Accepted 10 September 2018; Published 31 October 2018

Academic Editor: Bernabé L. Rivas

Copyright (c) 2018 Bin Zhang et al. This is an open access article distributed under the Creative Commons Attribution License, which permits unrestricted use, distribution, and reproduction in any medium, provided the original work is properly cited.

\begin{abstract}
Carboxylated polystyrene (PS) microspheres were synthesized by the two-step dispersion polymerization of styrene in hydrocarbon alcohols $\left(\mathrm{C}_{\mathrm{n}} \mathrm{H}_{2 \mathrm{n}+1} \mathrm{OH}, n=1-5\right)$ in the presence of acrylic acid (AA) as a functional comonomer, 2,2'-azobis(2-methylbutyronitrile) (AMBN) as the initiator and polyvinylpyrrolidone (PVP55) as the dispersant. The effects of solvent type, AA concentration, and first reaction time on the carboxyl content on the microsphere surfaces were investigated. The PS microspheres were characterized by scanning electron microscopy, infrared spectroscopy, nuclear magnetic resonance, and gel permeation chromatography. The results showed that the first reaction time of the two-stage dispersion polymerization had a great influence on the nucleation process, and appropriately prolonging the first-step reaction time had a great influence on the surface carboxyl content. The effect of the solvent on the surface carboxyl content of PS microspheres was significant. With n-butanol as the solvent, the carboxyl group content on the surface of the microspheres reached $57.05 \mathrm{mg} / \mathrm{g}$.
\end{abstract}

\section{Introduction}

In recent years, as the emphasis on health has increased, the technical requirements for the detection of various indicators in serum have become increasingly high. Dry chemical in vitro diagnostic reagents have the advantages of convenience, portability, accuracy, and no pollution $[1,2]$ and have attracted the attention of many researchers. A dry chemical in vitro diagnostic reagent usually consists of a diffusion layer, reagent layer, indicator layer, and substrate layer. After the tested sample diffuses through the diffusion layer and then reacts with the reagent layer, it is detected and converted into a signal value after the indicator layer is colored. Therefore, the diffusion effect of the diffusion layer determines the accuracy of the detection. Good diffusion results require fast and uniform diffusion. At present, dry chemical in vitro diagnostic reagents independently developed at home and abroad mostly utilize diffusion papers such as filter papers [3] and polysulfonic acid [4]. The diffusion layers using these types of materials cause inaccurate test results because of their poor diffusion and reactivity. In addition, the diffusion layer often has the function of immobilizing solid reagents and biological ligands. Therefore, the selection of materials with high surface reactivity and uniform diffusivity is necessary for the preparation of dry chemical in vitro diagnostic reagents.

Carboxylated polystyrene (PS) microspheres have high reactivity with various ligands, particularly biomolecules, and have widespread applications. The surface modification with carboxyl groups can improve the colloidal stability of the microsphere dispersion and prevent particle aggregation [5]. Carboxylated microspheres are often used in biomedical and diagnostic fields such as drug delivery, sensors, and biological detection. For example, polypyrrole-coated PS particles are used to adsorb proteins as a new diagnostic material [6-10].

There are many polymerization methods for preparing PS microspheres, such as precipitation polymerization, seed polymerization, microemulsion, and dispersion polymerization. 
Precipitation polymerization is a heterogeneous polymerization method, and the prepared particles have the advantages of low viscosity and clean surfaces but low solid contents and irregular geometries [11, 12]. Seed polymerization is a typical preparation method for core-shell emulsions. The particle size distribution obtained by this method is narrow, and the stability is good. However, the preparation process is cumbersome and complex, the period is long, and functional monomers easily become embedded in the particles [13]. Microemulsion is a method of forming an emulsion by using an emulsifier and forming particles in microbubbles; it can produce particles with uniform particle size and good stability, but the smaller particle size is still several nanometers [14]. In recent years, dispersion polymerization has gradually become the main method for synthesizing PS microspheres. Compared to other synthetic methods, dispersion polymerization has the advantages of easy functionalization, simple operation, uniform particle size, and an appropriate reaction period [15]. Song et al. used dispersion polymerization to discuss the effect of the amount of added acid on the carboxyl content on the surface of the microsphere [16] and summarized the effect of the amount of acid added on the appearance and surface carboxyl groups of microspheres, but they only discussed the solvent of ethanol. Lee and $\mathrm{Ha}$ et al. [17] used dispersion polymerization to prepare PS microspheres using fluorinated alcohol as the solvent; the synthesized microspheres had high carboxyl contents but small particle sizes. Bai et al. [18] prepared PS microspheres in an ionic liquid by dispersion polymerization, which expanded the types of solvents, but the sphericity was irregular and the dispersibility was poor. Many problems remain in dispersion polymerization, restricting the applications of carboxyl microspheres in the biomedical and diagnostic fields; these problems include low surface carboxyl content, nonuniform particle sizes, and irregular sphericity. The preparation of microspheres with regular spherical shapes and controllable carboxyl contents on the surface remains challenging.

In this study, carboxylated PS microspheres were prepared for use as the diffusion layers of in vitro diagnostic reagents to immobilize some curing reagents, such as phosphocholine. A two-step dispersion polymerization method was used to prepare carboxylated PS microspheres with controllable surface carboxyl groups by adjusting the solvent, the concentration of acrylic acid (AA) added, and the reaction time of the first step. The influence of the above preparation conditions on the surface carboxyl content of the microspheres was analyzed.

\section{Experiment}

2.1. Materials and Instrumentations. Styrene (St) was purchased from Sinopharm. Methanol, ethanol, propanol, butanol, and pentanol were purchased from Beijing Chemical Corporation. Polyvinylpyrrolidone (PVP, $\left.\mathrm{M}_{\mathrm{w}} \sim 55000\right)$, Triton (TX-100), acrylic acid (AA), and 2,2'-azobis-(2methylbutyronitrile) (AMBN) were purchased from J\&K Scientific Ltd. All of the reagents were used without further purification.
A Tensor-27 Fourier transform infrared spectroscopy (FTIR) made in Bruker (Germany) was utilized to measure the carboxyl group content on carboxylated polystyrene surface. A Zeiss-Supra 55 field emission scanning electron microscope (SEM) made in Carl Zeiss (Germany) was utilized at operation voltages from 2 to $5 \mathrm{kV}$ to observe particle morphology. The molecular weight was measured by the Waters-e2695 gel permeation chromatography (GPC) made in Waters (America) and the carboxyl group was measured by the Waters nuclear magnetic resonance (NMR) made in Waters (America).

2.2. Synthesis of Poly(St-co-AA) Microspheres. The microspheres were prepared by two-step dispersion polymerization. In the first step, the dispersant (PVP), codispersant (TX100), initiator (AMBN), monomer (St), and the solvent were added to a $250 \mathrm{~mL}$ four-necked reaction flask equipped with a nitrogen inlet, a thermometer, and a condenser. After a homogeneous solution was formed at room temperature, the solution was deoxygenated by bubbling nitrogen at room temperature for $10 \mathrm{~min}$. The flask was then placed in an oil bath at $70^{\circ} \mathrm{C}$ and subjected to mechanical stirring for $h$ $(\mathrm{h}=1-5)$ hour(s) at a rate of $180 \mathrm{rpm}$. In the second step, the AA was dissolved in a mixed solution of the equivalent St and the equivalent solvent at $70^{\circ} \mathrm{C}$ under nitrogen atmosphere. After the first reaction time, the acrylic solution was added to a four-necked flask. The reaction was stopped after $(24 \mathrm{~h})$ hours of continuous reaction. After the reaction was complete, the sample was ultrasonically dispersed in ethanol and centrifuged; the supernatant was discarded. After washing three times, the sample was stored in a centrifuge tube and sealed with ethanol to prepare for later use.

2.3. Characterization of Carboxylated PS Microspheres. The surface morphology and dispersion of microspheres were characterized by scanning electron microscopy (SEM, ZEISS-SUPRA55). The molecular weight of the microspheres was measured by high-performance gel permeation chromatography (GPC).

The surface carboxyl content of the microspheres was determined by the conductivity method. The mass of $m$ microspheres was weighted, ultrasonically dispersed in a sodium hydroxide solution of $25 \mathrm{mmol} / \mathrm{L}$ in concentration, and then put aside for 2 hours to allow full reaction of the carboxyl and sodium hydroxide. Then, the sample was titrated with $9 \mathrm{mmol} / \mathrm{L}$ hydrochloric acid solution to determine the change in conductivity. The conductivity decreased with the addition of hydrochloric acid solution, then tended to plateau, before increasing finally.

The conductivity declined with excessive sodium hydroxide. In this period, the hydrochloric acid and sodium hydroxide neutralization reaction occurred. The volume of consumed hydrochloric acid volume was denoted as $V_{1}$.

In the constant-conductivity phase of the hydrochloric acid and sodium carboxylate reaction, the volume of hydrochloric acid consumed is denoted as $V_{2}$.

The rising-conductivity phase is excessive in hydrochloric acid solution. 


$$
\begin{aligned}
& \text { Carboxyl content on surface }(\% / g)=\mathrm{M}_{\mathrm{COOH}} \times\left(\mathrm{V}_{2}-\mathrm{V}_{1}\right) \\
& \times \mathrm{C}_{\mathrm{HCl}} / \mathrm{m} \text {. }
\end{aligned}
$$

\section{Results and Discussion}

3.1. Carboxylation of Polystyrene Microspheres. Figure 1 is a NMR characterization diagram. It can be seen that the peak of $11 \mathrm{ppm}$ which is a characteristic peak of the hydrogen atom on the carboxyl group from the Figure 1 indicating that the carboxyl group is successfully modified to the surface of polystyrene. Figure 2 is a FTIR characterization diagram. The peak of $1700 \mathrm{~cm}-1$ which is a characteristic peak of the $\mathrm{C}=\mathrm{O}$ bond on the carboxyl group can also be seen. It indicates that the carboxyl group is successfully modified to the surface of polystyrene.

3.2. The Effect of First-Step Reaction Time on the Carboxyl Content of the Surface. Table 1 presents a table with all reaction conditions in this part for the synthesis experiments conducted.

Figure 3 shows that the carboxyl content on the surface of the microspheres first increases and then decreases with increases in the first-step reaction time, reaching a maximum at $3 \mathrm{~h}$. This is related to the nucleation process of the microspheres. When the initiator radicals and monomers are polymerized, the probability of polymerization of free radicals and monomers with high solubility is greater [19]. In the first step, the reaction time is short. There are many unreacted St monomers, and the formed microspheres are smaller in volume and quantity. In the second-step reaction, although the St monomer concentration is relatively high compared to that of AA and the probability of polymerization is greater, the acrylic monomer can still participate in the polymerization. At this time, the volume of the microspheres grows faster, and most of the carboxyl groups on the long chain of the polymer are embedded in the microspheres. As the reaction progresses, the diameter of the microspheres tends to increase gradually. At this time, the carboxyl groups on the long chain of the polymer are exposed on the surfaces of the microspheres. With the prolongation of the first-step reaction time, the St monomer added in the first step consumes more, the formed microspheres are larger in diameter and more in number, and the surface area is larger. In the second-step reaction, the newly generated long-chain carboxyl-containing polymer is more exposed to the surface of microspheres, and thus the surface carboxyl content increases. However, when the first-step reaction time is too long, the initiation efficiency of the initiator decreases, the active center decreases, the polymerization rate decreases, and the surface carboxyl content decreases. Therefore, as the first reaction time increases, the surface carboxyl content increases first and then decreases.

Figure 4 shows the Fourier transform infrared (FTIR) spectra of the PS microspheres prepared with different firststep reaction times. The respective absorption peaks are described in Table 2. The peak at $1700 \mathrm{~cm}^{-1}$ is a stretching vibration peak of the $\mathrm{C}=\mathrm{O}$ bond on the carboxyl group, characteristic of the carboxyl group. Compared to the characteristic peaks of PS adjacent to it (the stretching vibration

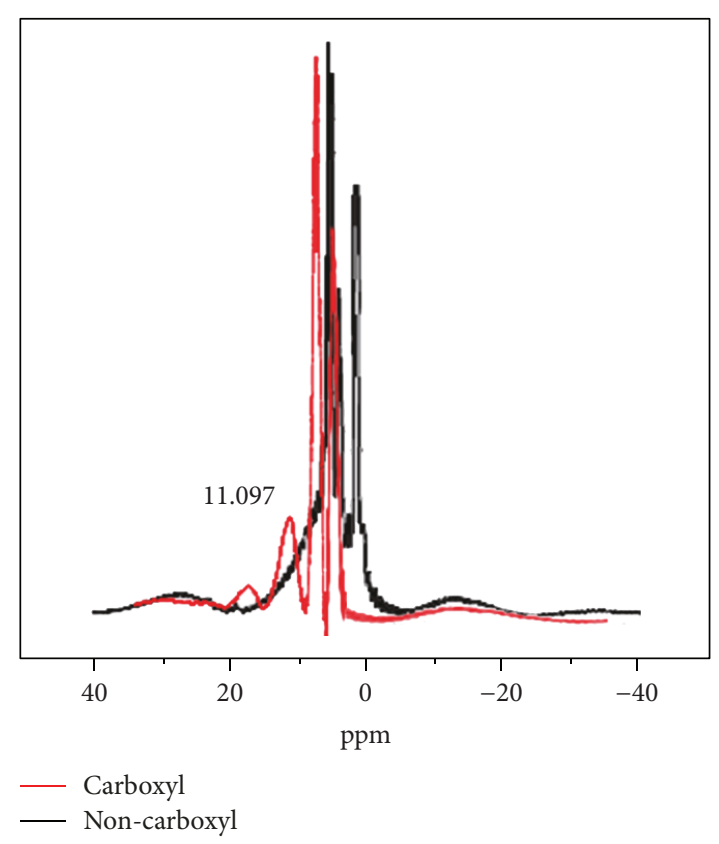

FIGURE 1: NMR spectra of carboxyl and noncarboxyl PS microspheres prepared.

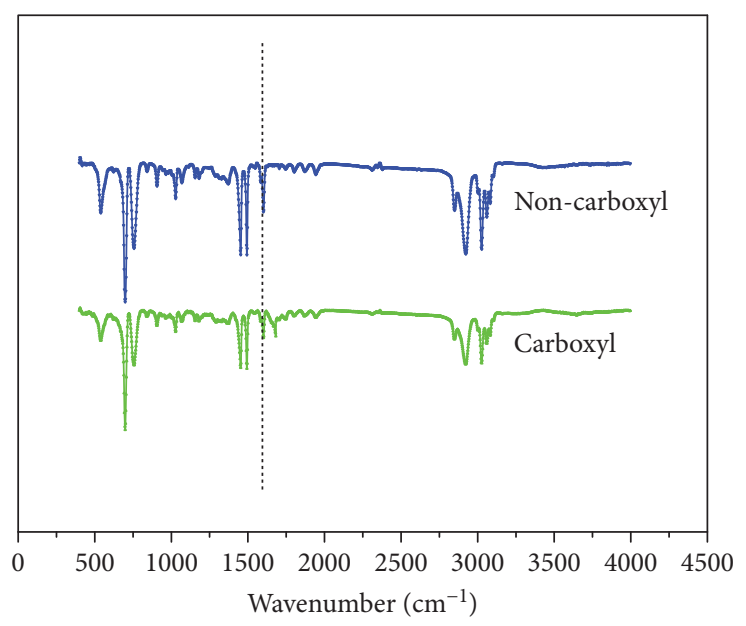

FIGURE 2: FTIR spectra of carboxyl and noncarboxyl PS microspheres prepared.

peaks at $1452 \mathrm{~cm}^{-1}, 1601 \mathrm{~cm}^{-1}$, and $1493 \mathrm{~cm}^{-1}$ on the benzene ring $-\mathrm{C}=\mathrm{C}-$ ), the intensity of the characteristic peak of the carboxyl group first increases and then decreases with increasing of the reaction time of the first step, reaching a maximum at $3 \mathrm{~h}$, which is consistent with the results obtained by the conductivity method.

3.3. Effect of Solvent on Carboxyl Content of the Surface. Table 3 presents a table with all reaction conditions in this part for the synthesis experiments conducted.

As can be seen from Figure 5, with the addition of an equal amount of acid, the carboxyl content on the surface of the microspheres is increased as the solvent carbon chain length increases. Xing [20] suggested that this was related to the polarity of the solvent. With increasing carbon chain 
TABLE 1: Synthesis experiment conditions in investigation of the effect of first-step reaction time on the carboxyl content of the surface.

\begin{tabular}{|c|c|c|c|c|c|c|}
\hline & Solvent & $\mathrm{m}_{\mathrm{PVP}}: \mathrm{m}_{\mathrm{St}}$ & $\mathrm{m}_{\mathrm{AMBN}}: \mathrm{m}_{\mathrm{S}}$ & St wt $\%$ & Reaction time of the first step & AA wt $\%$ \\
\hline 1 & Ethanol & 0.20 & 0.02 & $20 \%$ & $1 \mathrm{~h}$ & $7 \%$ \\
\hline 2 & Ethanol & 0.20 & 0.02 & $20 \%$ & $2 \mathrm{~h}$ & $7 \%$ \\
\hline 3 & Ethanol & 0.20 & 0.02 & $20 \%$ & $3 \mathrm{~h}$ & $7 \%$ \\
\hline 4 & Ethanol & 0.20 & 0.02 & $20 \%$ & $4 \mathrm{~h}$ & $7 \%$ \\
\hline 5 & Ethanol & 0.20 & 0.02 & $20 \%$ & $5 \mathrm{~h}$ & $7 \%$ \\
\hline
\end{tabular}

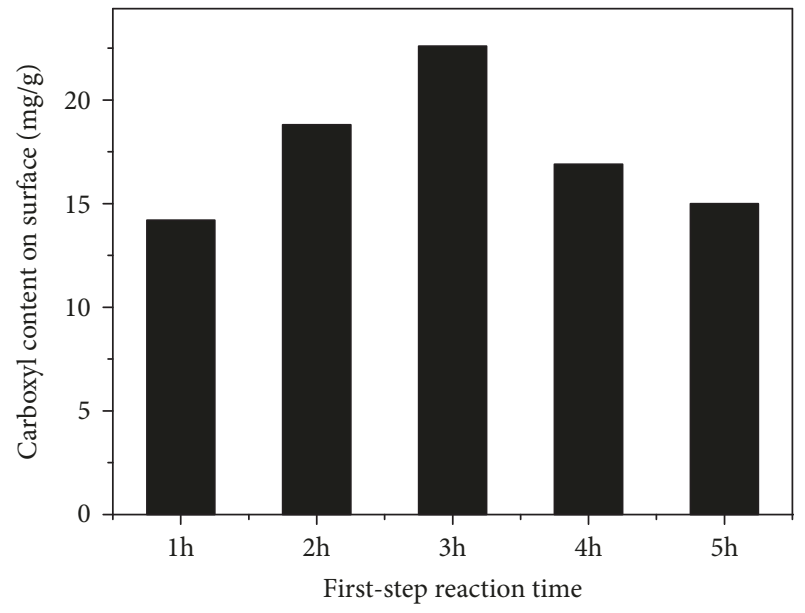

Figure 3: The effect of first-step reaction time on the carboxyl content on the surface (reaction conditions: $\mathrm{m}_{\mathrm{PVP}}: \mathrm{m}_{\mathrm{St}}=0.20$; $\mathrm{m}_{\mathrm{AMBN}}: \mathrm{m}_{\mathrm{St}}=0.02$; St $\mathrm{wt} \%=20 \%$; ethanol as solvent; AA wt $\%=7$ ).

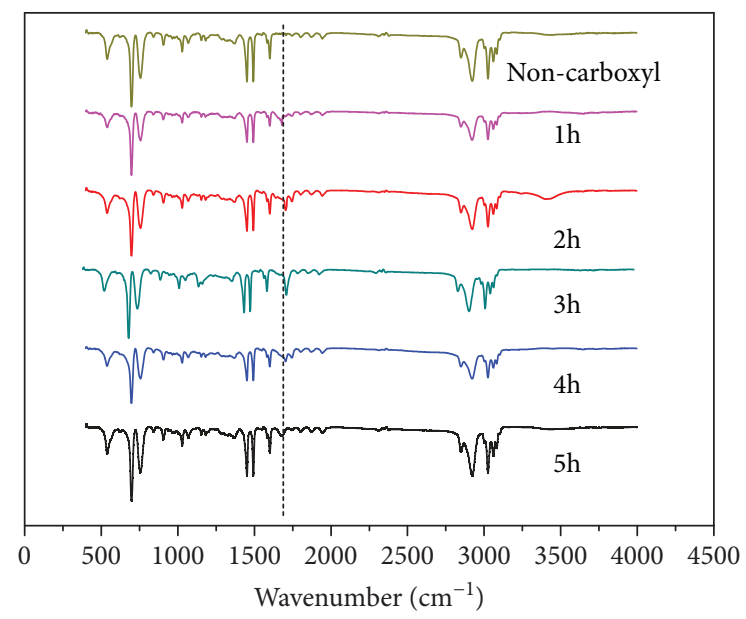

FIGURE 4: FTIR spectra of PS microspheres prepared with different times of the first step.

length in the solvent, the polarity of the solvent decreases, the solubility of the long chain of the polymer increases, and the critical length of the long chain of the polymer becomes longer, so that the size of the primary nucleus increases and the newly generated long-chain carboxyl-containing polymer is more exposed on the surface of microspheres, thus increasing the surface carboxyl content.

The results of the determination of the molecular weight and the molecular weight distribution of the PS microspheres are shown in Table 4. It demonstrates that, as the carbon
TABLE 2: Wavenumbers of functional groups in IR spectra of microspheres.

\begin{tabular}{|c|c|c|}
\hline Number & $\begin{array}{l}\text { Wavenumber } \\
\qquad\left(\mathrm{cm}^{-1}\right)\end{array}$ & Absorption peak \\
\hline 1 & 3100-3000 & $\begin{array}{l}\text { The stretching vibration peak of -C-H- } \\
\text { on benzene ring }\end{array}$ \\
\hline 2 & 2922 & $\begin{array}{l}\text { The stretching vibration peak of -C-H- } \\
\text { on saturated carbon }\end{array}$ \\
\hline 3 & 1703 & $\begin{array}{l}\text { The stretching vibration peak of the } \\
\mathrm{C}=\mathrm{O} \text { bond on the carboxyl group }\end{array}$ \\
\hline \multirow{3}{*}{4} & 1452 & \multirow{3}{*}{$\begin{array}{l}\text { The stretching vibration peak of }-\mathrm{C}=\mathrm{C}- \\
\text { on benzene ring }\end{array}$} \\
\hline & 1601 & \\
\hline & 1493 & \\
\hline
\end{tabular}

chain of the solvent is increased, the solubility of the solvent to the long chain of the polymer increases and the molecular weight of the polymer increases. It can be further speculated that larger polymer molecular weights correspond to larger formed primary nuclei, larger specific surface areas of the nuclei, and increased exposure of carboxyl groups; therefore, the carboxyl content on the microsphere surface increases with PS molecular weight.

Figure 6 is the FTIR spectra of carboxylated PS prepared in different solvents. From Figure 6, it can be seen that as the length of the carbon chain in the solvent increases, the intensity of the characteristic peak of the carboxyl group at $1703 \mathrm{~cm}^{-1}$ is increased, indicating that the carboxyl content on the surface of the microsphere is increased. This rule is consistent with the results of the conductivity method shown in Figure 5.

3.4. Effect of $A A$ Concentration on Carboxyl Content of Microspheres. Table 5 presents a table with all reaction conditions in this part for the synthesis experiments conducted.

Carboxylated PS microspheres were prepared in ethanol as the solvent, and the effect of the AA concentration on the carboxyl content on the surface of microspheres was investigated. When the concentration of $\mathrm{AA}$ is $7 \mathrm{wt} \%$ of the total amount of the monomers, microspheres with regular appearance are obtained as shown in Figure 7(a), and the surface carboxyl group content of the microspheres is $14.2 \mathrm{mg} / \mathrm{g}$ as measured by the conductivity method. When the AA concentration is increased to $10 \mathrm{wt} \%$, the carboxyl content on the surface of the microspheres increases to $17.1 \mathrm{mg} / \mathrm{g}$. When the AA concentration is increased to $14 \mathrm{wt} \%$ (the concentration limit for the preparation of PS microspheres), microspheres are not obtained; the product is a transparent mass, with 
TABle 3: Synthesis experiments conditons in investigation of the effect of solvent on carboxyl content of the surface.

\begin{tabular}{lcccccc}
\hline & Solvent & $\mathrm{m}_{\mathrm{PVP}}: \mathrm{m}_{\mathrm{St}}$ & $\mathrm{m}_{\mathrm{AMBN}}: \mathrm{m}_{\mathrm{S}}$ & St wt\% & Reaction time of the first step & AA wt $\%$ \\
\hline 1 & Methanol & 0.20 & 0.02 & $20 \%$ & $1 \mathrm{~h}$ & $1 \mathrm{~h}$ \\
2 & Ethanol & 0.20 & 0.02 & $20 \%$ & $1 \mathrm{~h}$ & $7 \%$ \\
3 & Propanol & 0.20 & 0.02 & $20 \%$ & $1 \mathrm{~h}$ & $7 \%$ \\
4 & Butanol & 0.20 & 0.02 & $20 \%$ & $1 \mathrm{~h}$ & $7 \%$ \\
5 & Pentanol & 0.20 & 0.02 & $20 \%$ & & $7 \%$ \\
\hline
\end{tabular}

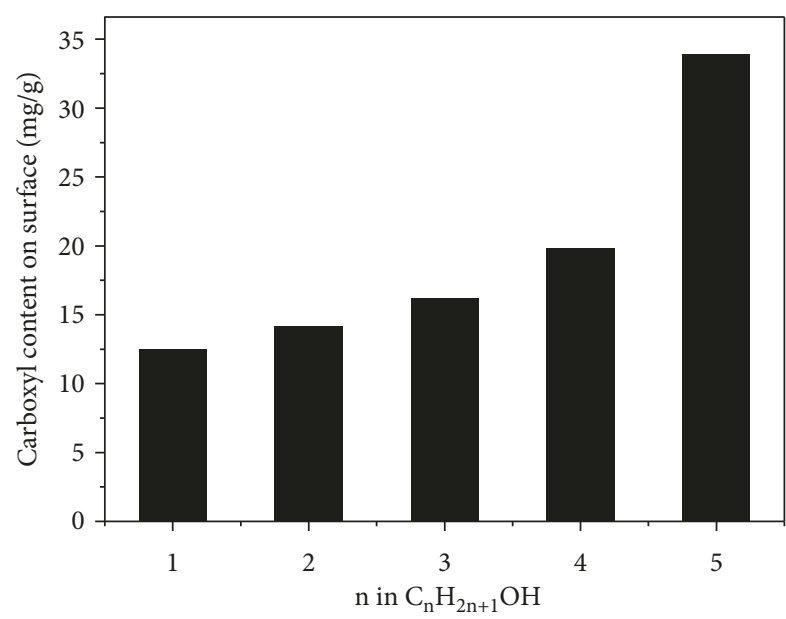

Figure 5: Effect of solvent on carboxyl content of the surface (reaction conditions: $\mathrm{m}_{\mathrm{PVP}}: \mathrm{m}_{\mathrm{St}}=0.20 ; \mathrm{m}_{\mathrm{AMBN}}: \mathrm{m}_{\mathrm{St}}=0.02 ;$ St wt $\%=$ $20 \%$; the first-step reaction time $=1 \mathrm{~h}$; AA wt $\%=7 \%$ ).

TABLE 4: Molecular weight of PS microspheres in different solvents.

\begin{tabular}{lccc}
\hline Solvent & $\mathrm{M}_{\mathrm{n}}$ & $\mathrm{M}_{\mathrm{w}}$ & $\mathrm{M}_{\mathrm{w}} / \mathrm{M}_{\mathrm{n}}$ \\
\hline Methanol & 99407 & 160244 & 1.612 \\
Ethanol & 116312 & 202382 & 1.740 \\
Propanol & 129899 & 246937 & 1.901 \\
Butanol & 225064 & 514496 & 2.286 \\
Pentanol & 460272 & 1168630 & 2.539 \\
\hline
\end{tabular}

microscopic morphology as shown in Figure 7(b). When the concentration of $\mathrm{AA}$ as a comonomer increases, the probability of the self-polymerization of the acrylic monomer increases; the influence of the polyacrylic acid (PAA) chain segment in the copolymer cannot be ignored. PAA has a greater affinity for ethanol than PS does; thus, increasing the content of PAA in the PS-PAA copolymer impedes product precipitation from the solvent. The polymerization system has a reaction limit in the concentration of AA. When the reaction limit is exceeded, the system becomes unstable, resulting in failure to yield spherical particles. Therefore, the effect of other solvents on the maximum concentration of AA in the solvent and the effect of AA on the surface carboxyl content in each solvent is examined.

Figure 8 shows the relationship between the surface carboxyl content of PS microspheres and the AA concentration in different solvents. From Figure 8, it can be seen that as

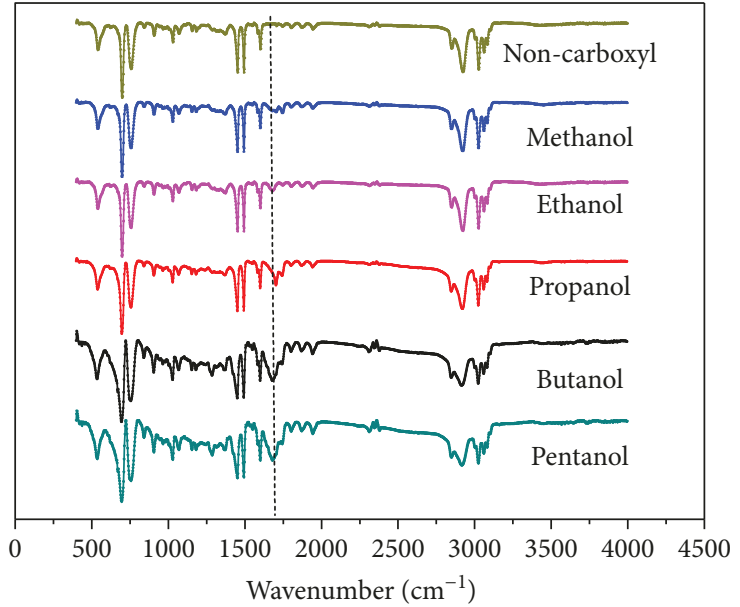

FIGURE 6: FTIR spectra of PS microspheres prepared in different solvents.

the concentration of AA is increased, the carboxyl content on the surface of the microspheres increases. With the same AA concentration, the surface carboxyl content of the microspheres is increased with the increasing of the carbon chain length in the solvent. This can be attributed to the polarity of the solvent. With the increasing of the carbon number in the solvent, the polarity decreases, and the affinity of the PAA segment in the PS-PAA copolymer decreases. This is favorable for the precipitation of the polymer from the solvent, resulting in the increase of the surface carboxyl content of the microspheres.

Figure 9 is an infrared spectrum of carboxylated polystyrene prepared in different solvents with the limit concentration of AA. From Figure 9, it can be seen that as the length of the carbon chain in the solvent increases, the intensity of the characteristic peak of the carboxyl group at $1703 \mathrm{~cm}-1$ increases first and then decreased, indicating that the carboxyl content on the surface of the microspheres increases first and then decreased.

Figure 10 shows the limit concentration of AA for the different solvents. If the limit concentration is exceeded, PS microspheres cannot be successfully prepared. As can be seen from Figure 10, the limit concentration of the AA for preparing the PS microspheres is maximized in butanol. For alcohol solvents with carbon numbers between one and four, the limit concentration of the AA concentration increases with increasing of the carbon number in the solvent. It is consistent with the results of the FTIR spectra shown in Figure 9. 
TABLE 5: Synthesis experiment conditions in investigation of effect of acrylic acid concentration on carboxyl content of microspheres.

\begin{tabular}{|c|c|c|c|c|c|c|}
\hline & Solvent & $\mathrm{m}_{\mathrm{PVP}}: \mathrm{m}_{\mathrm{St}}$ & $\mathrm{m}_{\mathrm{AMBN}}: \mathrm{m}_{\mathrm{S}}$ & St wt\% & Reaction time of the first step & AA wt $\%$ \\
\hline 1 & Methanol & 0.20 & 0.02 & $20 \%$ & $1 \mathrm{~h}$ & $7 \%$ \\
\hline \multirow{2}{*}{2} & \multirow{2}{*}{ Ethanol } & \multirow{2}{*}{0.20} & \multirow{2}{*}{0.02} & \multirow{2}{*}{$20 \%$} & \multirow{2}{*}{$1 \mathrm{~h}$} & $7 \%$ \\
\hline & & & & & & 10.5 \\
\hline \multirow{3}{*}{3} & \multirow{3}{*}{ Propanol } & \multirow{3}{*}{0.20} & \multirow{3}{*}{0.02} & \multirow{3}{*}{$20 \%$} & \multirow{3}{*}{$1 \mathrm{~h}$} & $7 \%$ \\
\hline & & & & & & $14 \%$ \\
\hline & & & & & & $21 \%$ \\
\hline \multirow{4}{*}{4} & \multirow{4}{*}{ Butanol } & \multirow{4}{*}{0.20} & \multirow{4}{*}{0.02} & \multirow{4}{*}{$20 \%$} & \multirow{4}{*}{$1 \mathrm{~h}$} & $7 \%$ \\
\hline & & & & & & $14 \%$ \\
\hline & & & & & & $21 \%$ \\
\hline & & & & & & $28 \%$ \\
\hline \multirow{2}{*}{5} & \multirow{2}{*}{ Pentanol } & \multirow{2}{*}{0.20} & \multirow{2}{*}{0.02} & \multirow{2}{*}{$20 \%$} & \multirow{2}{*}{$1 \mathrm{~h}$} & $7 \%$ \\
\hline & & & & & & $14 \%$ \\
\hline
\end{tabular}

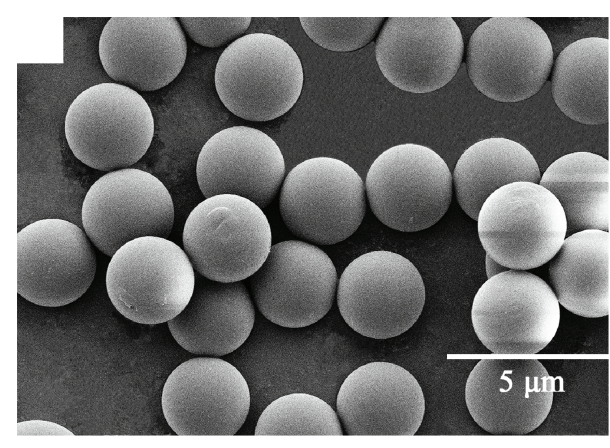

(a)

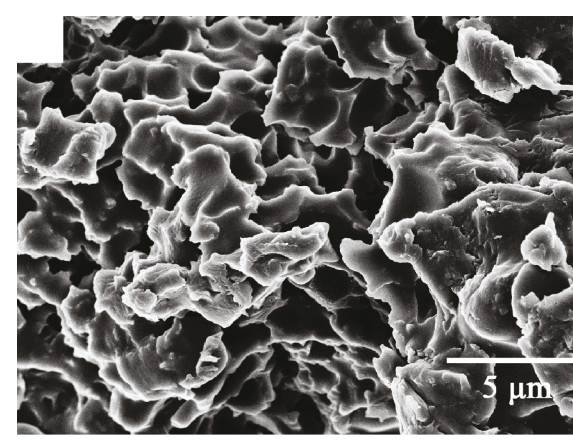

(b)

FIGURE 7: SEM micrographs of PS microspheres with different amount of AA (a). 7 wt\% AA; (b). 14 wt\% AA) (reaction conditions: $\mathrm{m}_{\mathrm{PVP}}: \mathrm{m}_{\mathrm{St}}=0.20 ; \mathrm{m}_{\mathrm{AMBN}}: \mathrm{m}_{\mathrm{St}}=0.02 ; \mathrm{St} \mathrm{wt} \%=20 \%$; the first-step reaction time $=1 \mathrm{~h}$; ethanol as solvent).

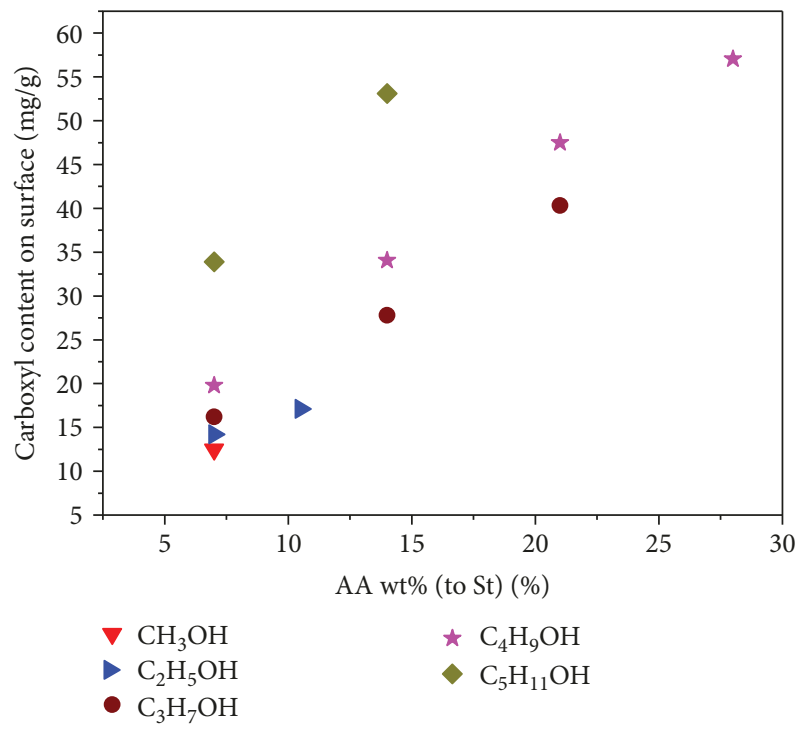

Figure 8: The effects of solvents on the surface carboxyl content (reaction conditions: $\mathrm{m}_{\mathrm{PVP}}: \mathrm{m}_{\mathrm{St}}=0.20 ; \mathrm{m}_{\mathrm{AMBN}}: \mathrm{m}_{\mathrm{St}}=0.02 ;$ St wt $\%=$ $20 \%$; the first-step reaction time $=1 \mathrm{~h}$ ).

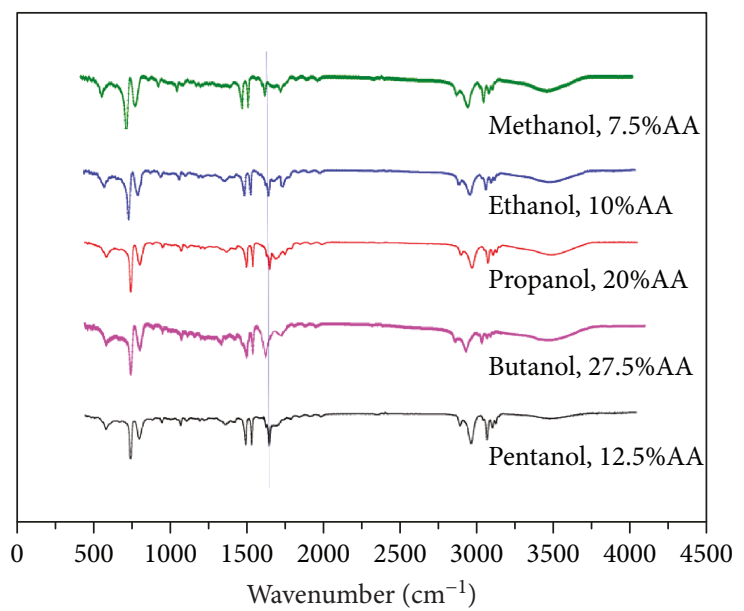

FIGURE 9: FTIR spectra of PS microspheres prepared in different solvents with limit concentration of acrylic acid.

This is also related to the polarity of the solvent; lower solvent polarity corresponds to the lower affinity for the PAA segment in the PS-PAA copolymer. It can be considered that 


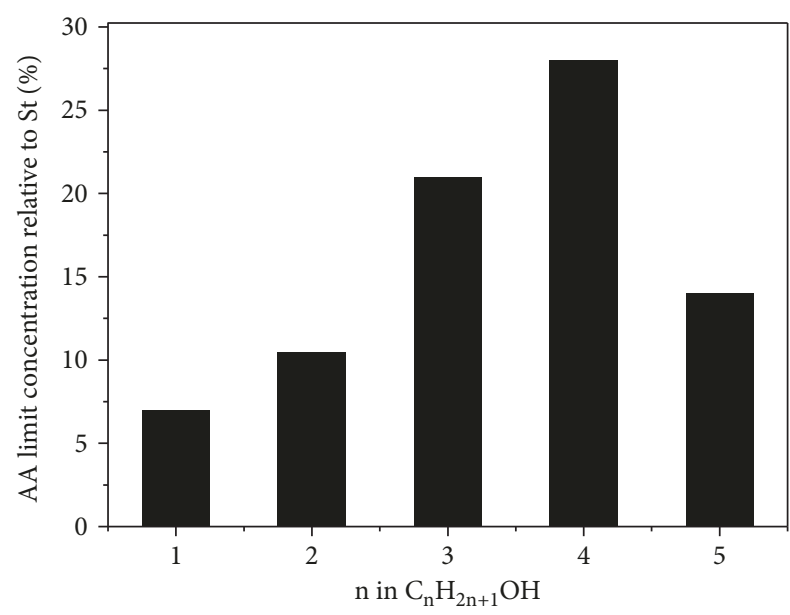

Figure 10: The limit concentration of AA for different solvents (reaction conditions: $\mathrm{m}_{\mathrm{PVP}}: \mathrm{m}_{\mathrm{St}}=0.20 ; \mathrm{m}_{\mathrm{AMBN}}: \mathrm{m}_{\mathrm{St}}=0.02 ; \mathrm{b} .20 \mathrm{wt} \%$; the first-step reaction time $=1 \mathrm{~h}$ ).

lower solvent polarity is related to the weakened influence of the PAA segment on the precipitation of the PS-PAA copolymer; thus, the PAA content can be further increased. Table 6 is the molecular weight of PS microspheres synthesized in different solvents with limit concentration of acrylic acid, and this result can also confirm the above conclusions.

Wang et al. [21] believed that, because of the ionization of carboxyl groups on the surface of the microspheres, the repulsion among microspheres was increased and the stability of the particles was enhanced. As the concentration of AA increased, the reaction rate of the entire system increased. When the concentration of AA reaches a certain value, a large amount of AA is polymerized onto the long chain, and excessive hydrophilic groups are introduced onto the polymer chain, thus prolonging the nucleation period. At the same time, the particle surface becomes sticky because of the tendency of the medium to dissolve. It is easy to coalesce; when the AA concentration is too high, maintaining sphericity in the product is difficult.

\section{Conclusion}

Carboxylated PS microspheres are prepared by a two-step dispersion method using C1-C5 hydrocarbon alcohols as the solvents. By changing the process conditions, the surface carboxyl content of the PS microspheres can be well controlled.

The effect of the first-step reaction time, solvent, and AA concentration on the carboxyl content on the surface of PS microspheres is investigated. The results show that the effect of solvent on the carboxyl content of the PS microspheres is significant. When $\mathrm{n}$-butanol is used as the solvent, the carboxyl content of PS microspheres can reach $57.05 \mathrm{mg} / \mathrm{g}$. The first-step reaction time of the two-step method also has some influence on the carboxyl content on the surface of the microspheres, mainly reflected in the influence of the first reaction time on the nucleation process.
TABLE 6: Molecular weight of PS microspheres in different solvents with limit concentration of AA.

\begin{tabular}{lcccc}
\hline Solvent & AA (\%) & $\mathrm{M}_{\mathrm{n}}$ & $\mathrm{M}_{\mathrm{w}}$ & $\mathrm{M}_{\mathrm{w}} / \mathrm{M}_{\mathrm{n}}$ \\
\hline Methanol & 7.5 & 99902 & 166244 & 1.664 \\
Ethanol & 10 & 135810 & 265780 & 1.957 \\
Propanol & 20 & 174992 & 385507 & 2.203 \\
Butanol & 27.5 & 371524 & 1563001 & 4.207 \\
Pentanol & 12.5 & 502101 & 1226633 & 2.443 \\
\hline
\end{tabular}

Carboxylated PS microspheres have important applications in biomedical detection, and achieving controllable surface carboxyl contents for microspheres establishes a good foundation for advancing their application in biomedical dry slides.

\section{Data Availability}

The data used to support the findings of this study are available from the corresponding author upon request.

\section{Conflicts of Interest}

The authors declare that they have no conflicts of interest.

\section{Acknowledgments}

This research is supported by the National Natural Science Foundation of China (91634101, 21601016), the Beijing Municipal Natural Science Foundation (2174073), and the Project of Construction of Innovative Teams and Teacher Career Development for Universities and Colleges under Beijing Municipality (IDHT20180508).

\section{References}

[1] P. Lapekas, "New technologies for laboratory productivity," Journal of Healthcare Materiel Management, vol. 12, no. 3, pp. 30-32, 1994.

[2] R. H. Ng, K. M. Sparks, and B. E. Statland, "Colorimetric determination of potassium in plasma and serum by reflectance photometry with a dry-chemistry reagent," Clinical Chemistry, vol. 38, no. 7, pp. 1371-1372, 1992.

[3] Y. H. Yu, H. Y. Yang, X. Zhang, Y. H. Li, and Y. Y. Niu, "Monolayer dry film for renal function examination and preparation method of monolayer dry film," China Patent CN104020283A, 2014.

[4] A. Yoshihiko, M. Kishi, and I. Hiroko, "Method for measuring high density lipoprotein cholesterol," Japan Patent CN101078729A, 2007.

[5] J. Jacobs, A. Byrne, N. Gathergood, T. E. Keyes, J. P. A. Heuts, and A. Heise, "Facile synthesis of fluorescent latex nanoparticles with selective binding properties using amphiphilic glycosylated polypeptide surfactants," Macromolecules, vol. 47, no. 21, pp. 7303-7310, 2014.

[6] P. M. Dietrich, A. Hennig, M. Holzweber et al., "Surface analytical study of poly(acrylic acid)-grafted microparticles (beads): characterization, chemical derivatization, and 
quantification of surface carboxyl groups," The Journal of Physical Chemistry C, vol. 118, no. 35, pp. 20393-20404, 2014.

[7] S. Bousalem, A. Yassar, T. Basinska et al., "Synthesis, characterization and biomedical applications of functionalized polypyrrole-coated polystyrene latex particles," Polymers for Advanced Technologies, vol. 14, no. 11-12, pp. 820-825, 2003.

[8] R. H. Utama, M. Drechsler, S. Forster, P. B. Zetterlund, and M. H. Stenzel, "Synthesis of $\mathrm{pH}$-responsive nanocapsules via inverse miniemulsion periphery RAFT polymerization and post-polymerization reaction," ACS Macro Letters, vol. 3, no. 9, pp. 935-939, 2014.

[9] X. Liu, Y. Xu, S. Ma et al., "Encapsulation of quantum dot clusters in stimuli-responsive spherical polyelectrolyte brushes," Industrial \& Engineering Chemistry Research, vol. 53, no. 28, pp. 11326-11332, 2014.

[10] J. Sun, C. Yi, W. Wei, D. Zhao, Q. Hu, and X. Liu, "Nanohybrids from direct chemical self-assembly of poly(styrene-altmaleic anhydride) as $\mathrm{pH}$-responsive particulate emulsifiers," Langmuir, vol. 30, no. 49, pp. 14757-14764, 2014.

[11] R. Perrier-Cornet, V. Heroguez, A. Thienpont, O. Babot, and T. Toupance, "Functional crosslinked polymer particles synthesized by precipitation polymerization for liquid chromatography," Journal of Chromatography A, vol. 1179, no. 1, pp. 2-8, 2008.

[12] T. J. Romack, E. E. Maury, and J. M. DeSimone, "Precipitation polymerization of acrylic acid in supercritical carbon dioxide," Macromolecules, vol. 28, no. 4, pp. 912-915, 1995.

[13] D. Wang and Y. Liu, "Mono-dispersed cross-linked polystyrene micro-spheres prepared by seed swelling polymerization method," Frontiers of Chemistry in China, vol. 3, no. 4, pp. 440-444, 2008.

[14] X. Zhao, S. Zhou, M. Chen, and L. Wu, "Effective encapsulation of Sudan Black B with polystyrene using miniemulsion polymerization," Colloid and Polymer Science, vol. 287, no. 8, pp. 969-977, 2009.

[15] W. Yang, D. Yang, J. Hu, C. Wang, and S. Fu, "Dispersion copolymerization of styrene and other vinyl monomers in polar solvents," Journal of Polymer Science Part A: Polymer Chemistry, vol. 39, no. 4, pp. 555-561, 2001.

[16] J. S. Song, L. Chagal, and M. A. Winnik, "Monodisperse micrometer-size carboxyl-functionalized polystyrene particles obtained by two-stage dispersion polymerization," Macromolecules, vol. 39, no. 17, pp. 5729-5737, 2006.

[17] S. G. Lee and J.-W. Ha, "Synthesis of highly carboxylated monodisperse polystyrene microspheres by dispersion polymerization in fluorinated alcohol," Macromolecular Research, vol. 24, no. 8, pp. 675-683, 2016.

[18] F. Bai, X. Yang, R. Li, B. Huang, and W. Huang, "Monodisperse hydrophilic polymer microspheres having carboxylic acid groups prepared by distillation precipitation polymerization," Polymer, vol. 47, no. 16, pp. 5775-5784, 2006.

[19] X. Hu, Preparation of carboxyl-functional polystyrene microspheres and its application in emulsion polymerization, [M.S. thesis], University of Science and Technology of China, 2009.

[20] Q. L. Xing, Preparation of monodisperse micron-sized pplystyrene microspheres and its self-assembly, Harbin Institute of Technology, 2012.

[21] Y. J. Wang, J. F. Liu, Z. L. Yao, C. D. Yuan, and T. Y. Cao, "Preparation and study of magnetic poly (methyl-methacrylate) microspheres," Journal of Tianjin University(Science and Technology), vol. 34, no. 1, pp. 64-68, 2001. 


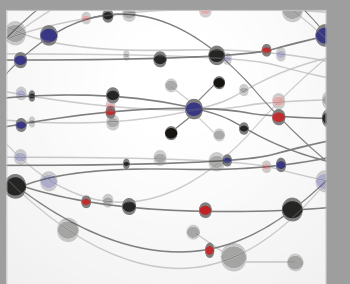

The Scientific World Journal
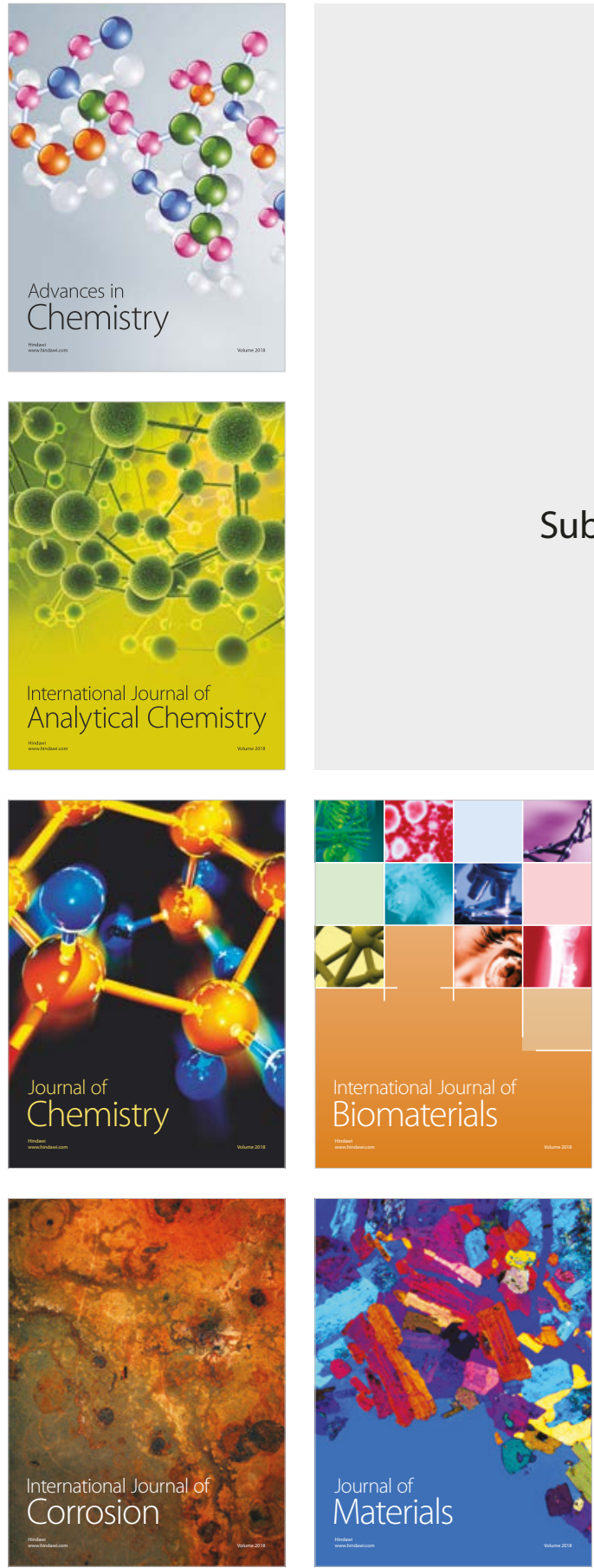

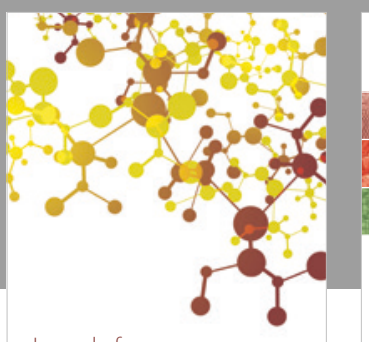

Journal of

Applied Chemistry
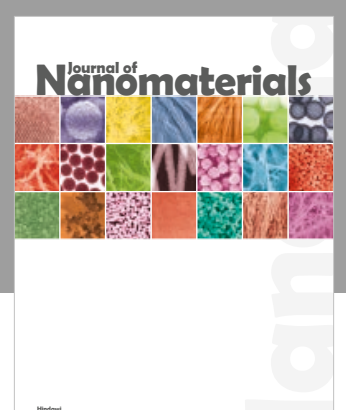

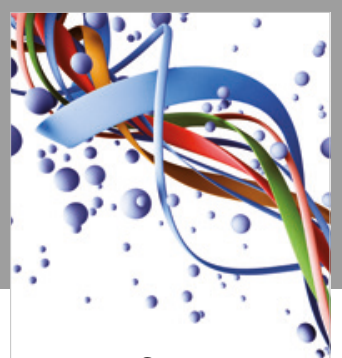

Scientifica

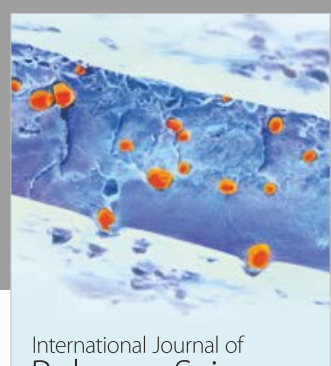

Polymer Science

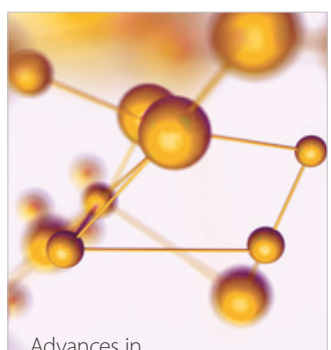

Physical Chemistry
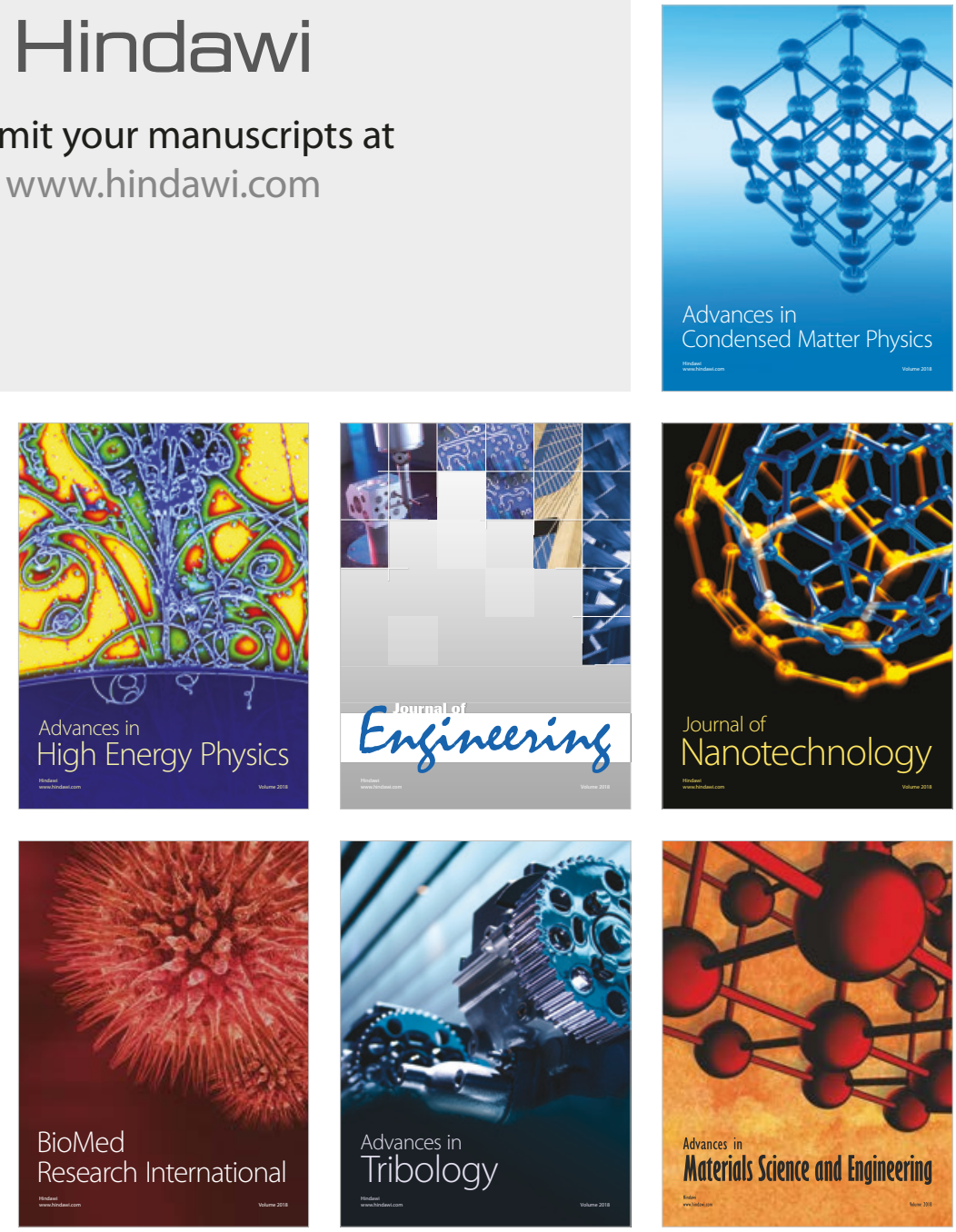